\title{
FLUTUAÇÃO POPULACIONAL DE ANASTREPHA FRATERCULUS (WIEDEMANN) E CERATITIS CAPITATA (WIEDEMANN) (DIPTERA, TEPHRITIDAE) EM POMARES DE PESSEGUEIRO EM PORTO ALEGRE, RIO GRANDE DO SUL
}

\author{
Flávio Roberto Mello Garcia ${ }^{1}$ \\ Elio Corseuil $^{2}$
}

\begin{abstract}
POPULATION FLUCTUATION OF ANASTREPHA FRATERCULUS (WIEDEMANN) AND CERATITIS CAPITATA (WIEDEMANN) (DiPTERA, TEPHRITIDAE) IN PEACH ORCHARDS IN Porto Alegre, Rio Grande do Sul. This paper presents the study of population fluctuation of Anastrepha fraterculus (Wiedemann, 1824) and Ceratitis capitata (Wiedemann, 1830) in peach orchards in Porto Alegre city. The peak for A. fraterculus was in November and December and for $C$. capitata in December and January. There was no significant difference among the population levels in the cultivars Fla 13-72, Premier and Marli.

KEY WORDS. Diptera, Tephritidae, Anastrepha fraterculus, Ceratitis capitata, population fluctuation, fruit flies
\end{abstract}

O pessegueiro, Prunus persica (Linnaeus) (Rosaceae), é atacado por insetos nas diversas fases do desenvolvimento, predominantemente por espécimes incluídos em Coleoptera e Diptera (GALlo et al. 1988). Os dípteros pertencentes a Tephritidae constituem um grupo importante devido aos grandes prejuízos que causam à fruticultura (COSTA 1958; BRESSAN \& TELES 1991). O pêssego é uma das espécies vegetais mais suscetiveis ao ataque de moscas-das-frutas (MATIOLI et al. 1989). A espécie Anastrepha fraterculus (Wiedemann, 1830) é a praga primária mais abundante e importante nas fruteiras no sul do Brasil, atacando frutas nas diversas estações do ano. (BlEICHER et al. 1982; SAlles 1991).

Infestações extremamente baixas ou raras de Ceratitis capitata (Wiedemann, 1824) na Costa Rica em hospedeiros geralmente preferidos por esta mosca sugere a competição com espécies de Anastrepha (CHRISTENSON \& FOOTE 1960).

BLEICHER et al. (1978) estudando a flutuação populacional de $A$. fraterculus no município de Videira, Santa Catarina, constataram que mais de $61 \%$ das moscas foram capturadas de novembro a janeiro, sendo os picos populacionais em novembro e dezembro.

1) Departamento de Ciências Biológicas e da Saúde, Universidade do Oeste de Santa Catarina. Rua Senador Attílio Fontana 591-E, Caixa Postal 747, 89809-000 Chapecó, Santa Catarina, Brasil.

E-mail: flaviog@unoesc.rct-sc.br

2) Departamento de Biologia, Pontifícia Universidade Católica do Rio Grande do Sul. Avenida Ipiranga 6681, Caixa Postal 1429, 90619-000 Porto Alegre, Rio Grande do Sul, Brasil.

Revta bras. Zool. 15 (1): 153 - 158, 1998 
FEHN (1981) em coletas na Região Metropolitana de Curitiba e Irati, no Paraná em pomares de pessegueiro através de caça-moscas e manutenção de frutos, constatou que $A$. fraterculus e $C$. capitata foram as espécies mais abundantes e que o período de maior ataque destas espécies ocorreu em cultivares de amadurecimento mediano, semi-tardio e tardio.

LORENZATO (1988) relata que os níveis populacionais de ocorrência de $A$. fraterculus foram superiores a $97 \%$, entre as demais espécies do gênero coletadas em frutíferas rosáceas no sul do Brasil, contudo ressalta que em determinados períodos, no município de Porto Alegre, C. capitata chega a suplantar A. fraterculus.

MATioli et al. (1988) em estudo de suscetibilidade de cultivares de pêssego às moscas-das-frutas na região de Caldas - Minas Gerais, constatou que: 1) a espécie dominante foi $C$. capitata; 2) as moscas foram coletadas somente no período de outubro de 85 a fevereiro de 86 e o pico populacional ocorreu na última semana de dezembro de $86 ; 3$ ) algumas características varietais (coloração, pilosidade, maturação e consistência da polpa) do pessegueiro podem afetar o comportamento das moscas em relação à sua preferência por cultivares com frutos de cor amarela, consistência da polpa firme e pilosidade mediana.

SAlles \& Kovaleski (1990) constataram, que em mais de dez anos de captura de adultos em pomares de macieira e pessegueiro, há predominância de $A$. fraterculus e que a ocorrência é constante de início de novembro a final de janeiro, com o pico ocorrendo em meados de dezembro.

HICKEL \& DUCROQUET (1993) constataram que tanto em pomares de pêssego e de ameixa, o pico de moscas-das-frutas do gênero Anastrepha ocorreu de meados a fim de dezembro em Santa Catarina e que o número de moscas coletadas durante o período de maturação da cultivar Premier foi relativamente baixo, quando comparada com a Chiripá e Coral, visto ser mais precoce.

SAlLES (1994) em estudo de períodos de ataque de A. fraterculus em cinco cultivares de pessegueiro constatou que, a partir de determinada fase, ocorre um aumento progressivo na infestação, sendo as datas de início de infestação e de nível de risco máximo para cada cultivar respectivamente em Pelotas, RS: Precocinho: 23 e 30 de outubro; Ágata e Diamante: 12 e 19 de novembro; Br-2: 6 -7 e 17 de dezembro e para Eldorado estas datas ficaram entre 10 e 13 de dezembro.

Com o objetivo de listar as espécies de tefritídeos ocorrentes em pessegueiro em Porto Alegre, Rio Grande do Sul e determinar sua flutuação populacional realizou-se este trabalho.

\section{MATERIAL E MÉTODOS}

\section{Coleta de adultos em frascos caça-moscas}

Para coleta de adultos foram instaladas armadilhas caça-moscas em dois pomares da cultivar Fla 13-72, dois da Premier e dois da Marli, com um hectare cada, em Porto Alegre, Rio Grande do Sul. As armadilhas consistiam de cilíndros plásticos opacos de $21 \mathrm{~cm}$ de comprimento com $8 \mathrm{~cm}$ de diâmetro com quatro orifícios de $1 \mathrm{~cm}$ de diâmetro dispostos simetricamente a uma altura de $15 \mathrm{~cm}$, 
distanciados um frasco do outro por aproximadamente $10 \mathrm{~m}$, com $200 \mathrm{ml}$ de solução aquosa de vinagre de vinho tinto a $25 \%$ como atrativo, colocadas a uma altura aproximada de $1 \mathrm{~m} 50 \mathrm{~cm}$, dentro da copa de árvores de pessegueiro.

Os frascos, cinco por pomar, foram utilizados na captura de moscas-das-frutas no período de outubro de 1994 a setembro de 1995. Durante este período foram realizadas visitas semanais aos pomares para troca da solução atrativa e coleta dos tefritídeos, colocando-os em frascos etiquetados, contendo álcool $70 \%$ para posterior separação por sexo, contagem e identificação em laboratório.

\section{Identificação taxonômica}

Ceratitis capitata foi reconhecida por comparação com materiais contidos nas coleções do Museu de Ciências e Tecnologia da Pontifícia Universidade Católica do Rio Grande do Sul (PUCRS), e também utilizando as características diagnósticas apresentadas por ZUCCHI et al. (1993).

O reconhecimento de Anastrepha fraterculus foi baseado no ápice do ovipositor das fêmeas, para tanto, algumas fêmeas foram colocadas em posição ventral em uma lâmina sob microscópio estereoscópico, e com auxílio de dois estiletes foi extrovertido o ovipositor. O exame do ovipositor foi feito sob aumento de 40 ou 100 vezes, neste caso, se colocou uma gota de glicerina sobre ovipositor. Posteriormente foi usada a chave dicotômica para espécies de Anastrepha ocorrentes no Brasil.

Foram depositados exemplares de cada espécie coletada no Museu de Ciências e Tecnologia da PUCRS.

\section{Flutuação populacional e comparação com o ciclo da cultura}

Foram feitas anotações sobre o desenvolvimento do vegetal, tais como: época de floração e maturação dos frutos. Estas informações serviram para estabelecer comparação entre a fenologia da cultura e flutuação populacional dos tefritídeos.

Em função do número de adultos coletados foi feito cálculo de análise de variância com o uso de programa SANEST em bifatorial, sendo as cultivares o fator $\mathrm{A}$ e as épocas o fator $\mathrm{B}$, com duas repetições, em delineamento completamente casualizado. As médias foram agrupadas pelo teste de Duncan a $5 \%$ de probabilidade. A análise foi feita com os valores transformados em raiz quadrada de $(x+0,5)$.

Fez-se análise de variância para média de moscas/semanas dentro de cada mês de coleta visando a execução de cálculos com números mais expressivos de exemplares e para permitir valores a serem comparados com resultados de outros autores que se encontram dessa forma.

\section{RESULTADOS E DISCUSSÃO}

Através das análises de variância (Tab. I) verifica-se que houve significância para os meses de amostragem para ambos os sexos de Anastrepha fraterculus e seu total, para as fêmeas de Ceratitis capitata, para o total de fêmeas e para a comunidade de tefritídeos. 
Tabela I. Súmula da análise de variância dos números de Anastrepha fraterculus (1) e Ceratitis capitata (2) coletadas com frascos caça-moscas em pomares de pessegueiro em função dos meses, com valores transformados em raiz quadrada de $(x+0,5)$. Porto Alegre, Rio Grande do Sul, 1994/95.

\begin{tabular}{lccccc}
\hline Causas da variação & Cultivar & Meses & Interação & Residuo & C.V. \\
\hline \multicolumn{1}{c}{ G.L. } & 2 & 11 & 22 & 36 & - \\
\hline (1) Fêmea & 0,082 & $1,351^{*}$ & 0,099 & 0,989 & 29,1 \\
(1) Macho & 0,074 & $1,009^{*}$ & 0,090 & 0,136 & 36,5 \\
(1) Total & 0,097 & $2,861^{*}$ & 0,197 & 0,238 & 38,5 \\
(2) Fêmea & 0,098 & $0,277^{*}$ & 0,056 & 0,072 & 31,7 \\
(2) Macho & 0,007 & $0,015^{*}$ & 0,004 & 0,005 & 9,3 \\
(2) Total & 0,062 & $0,341^{*}$ & 0,059 & 0,083 & 33,6 \\
\hline Total de fêmeas & 0,176 & $1,762^{*}$ & 0,161 & 0,153 & 33,6 \\
Total de tefritídeos & 0,137 & $3,333^{*}$ & 0,270 & 0,300 & 40,5 \\
\hline
\end{tabular}

(*) Significância ao nivel de 5\%; (C.V.) Coeficiente de variação; (G.L.) Graus de liberdade.

As comparações entre as médias nos diversos meses encontram-se na tabela II.

Tabela II. Agrupamento das médias do número de Anastrepha fraterculus (1) e Ceratitis capitata (2)/semanas dentro de cada mês, coletadas com frascos caça-moscas em pomares de pessegueiro. Porto Alegre, Rio Grande do Sul, 1994/95.

\begin{tabular}{|c|c|c|c|c|c|c|c|c|}
\hline \multirow{2}{*}{ Meses } & \multicolumn{3}{|c|}{ (1) } & \multicolumn{3}{|c|}{ (2) } & \multirow{2}{*}{$\begin{array}{l}\text { Total de } \\
\text { - fêmeas }\end{array}$} & \multirow{2}{*}{$\begin{array}{c}\text { Total de } \\
\text { tefritídeos }\end{array}$} \\
\hline & Fêmea & Macho & Total & Fêmea & Macho & Total & & \\
\hline Outubro & $1,437 b$ & $1,147 b$ & $1,680 b$ & $0,707 c$ & $0,707 b$ & $0,707 \mathrm{c}$ & $1,438 b$ & $1,680 b$ \\
\hline Novembro & $2,086 a$ & $1,918 a$ & $2,750 a$ & $0,925 b c$ & $0,733 b$ & $0,947 b c$ & $2,179 a$ & $2,829 a$ \\
\hline Dezembro & $1,924 a$ & $1,780 a$ & $2,529 a$ & $1,393 a$ & $0,786 b$ & $1,435 a$ & $2,281 a$ & $2,821 a$ \\
\hline Janeiro & $0,879 c$ & $0,947 b$ & $1,072 b c$ & $1,149 a b$ & $0,876 a$ & $1,253 \mathrm{ab}$ & $1,271 b c$ & $1,512 b c$ \\
\hline Fevereiro & $0,756 c$ & $0,733 b$ & $0,775 c$ & $0,775 c$ & $0,733 b$ & $0,793 c$ & $0,810 \mathrm{~cd}$ & $0,839 \mathrm{~cd}$ \\
\hline Março & $0,802 c$ & $0,809 b$ & $0,900 c$ & $0,756 c$ & $0,707 b$ & $0,756 c$ & $0,836 \mathrm{~cd}$ & $0,934 \mathrm{~cd}$ \\
\hline Abril & $0,919 c$ & $0,900 \mathrm{~b}$ & $1,074 b c$ & $0,775 c$ & $0,707 b$ & $0,775 c$ & $0,969 \mathrm{bcd}$ & $1,120 \mathrm{bcd}$ \\
\hline Maio & $0,865 c$ & $0,812 b$ & $0,955 c$ & $0,750 c$ & $0,707 b$ & $0,750 \mathrm{c}$ & $0,898 \mathrm{~cd}$ & $0,999 \mathrm{bcd}$ \\
\hline Junho & $0,756 c$ & $0,760 \mathrm{~b}$ & $0,802 c$ & $0,707 c$ & $0,707 b$ & $0,707 c$ & $0,756 \mathrm{~cd}$ & $0,802 \mathrm{~cd}$ \\
\hline Julho & $0,802 c$ & $0,760 \mathrm{~b}$ & $0,846 c$ & $0,707 c$ & $0,707 b$ & $0,707 \mathrm{c}$ & $0,802 \mathrm{~cd}$ & $0,846 \mathrm{~cd}$ \\
\hline Agosto & $0,707 \mathrm{c}$ & $0,707 b$ & $0,707 c$ & $0,707 c$ & $0,707 b$ & $0,707 c$ & $0,707 d$ & $0,707 d$ \\
\hline Setembro & $0,994 c$ & $0,851 b$ & $1,104 b c$ & $0,760 c$ & $0,733 b$ & $0,782 c$ & $1,036 \mathrm{bcd}$ & $1,157 \mathrm{bcd}$ \\
\hline
\end{tabular}

${ }^{*}$ ) Médias seguidas da mesma letra, em cada coluna, não diferem pelo teste de Duncan ao nivel de $5 \%$

A maior ocorrência de A. fraterculus, tanto de fêmeas e machos como para o total, foi em novembro e dezembro (Fig. 1), concomitantemente com a frutificação das cultivares Premier e Marli, respectivamente. Resultados semelhantes foram obtidos por Bleicher et al. (1978), SALLES \& KovalesKi (1990) e Hickel \& DUCROQUET (1993). 

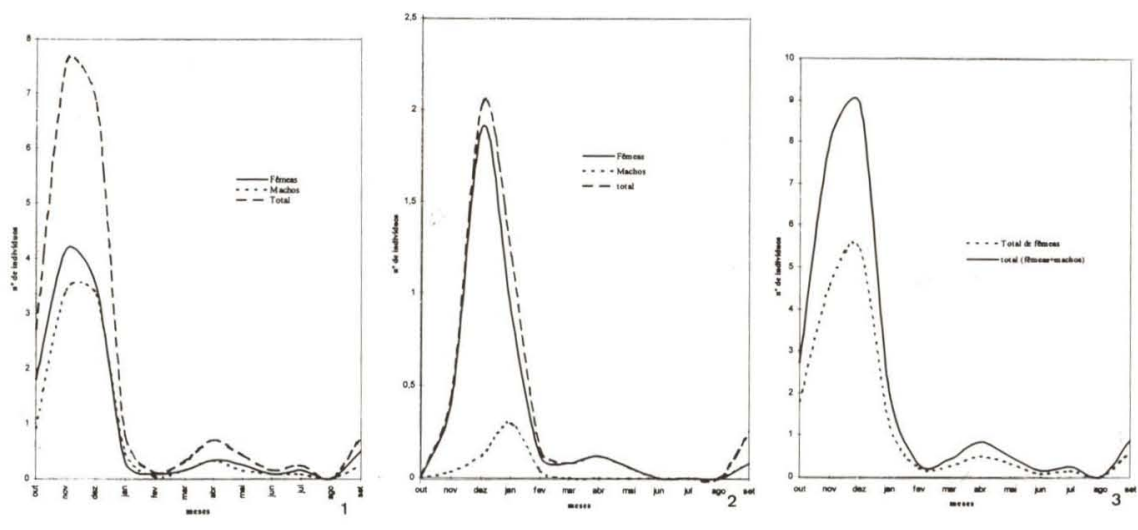

Figs 1-3. Flutuação populacional em pessegueiro, de outubro de 1994 a setembro de 1995 , Porto Alegre, Rio Grande do Sul. (1) Anastrepha fraterculus; (2) Ceratitis capitata; (3) A. fraterculus e C. capitata.

Para C. capitata, fêmeas e o total, o pico populacional foi em dezembro e janeiro corroborando os resultados obtidos por MATIOLI et al. (1988); para os machos (Fig. 2) foi somente em janeiro.

As fêmeas e a comunidade dos tefritídeos tiveram sua maior ocorrência em novembro e dezembro (Fig. 3).

As moscas-das-frutas estiveram presentes em todas as estações do ano tendo maior ocorrência na primavera e verão concordando com os dados obtidos por SALLES (1991) em Pelotas, Rio Grande do Sul.

A pequena elevação ocorrida na população no mês de abril deve-se provavelmente à época de maturação da goiaba, servindo essa de repositório da população.

Em todas as análises feitas houve significância apenas para as épocas de coleta, diferindo dos trabalhos de FEHN (1981); HiCKEL \& DUCROQUET (1993) e SALLES (1994) que ressaltam diferenças entre as cultivares; todavia estes autores avaliaram outro conjunto de cultivares.

Em nenhuma amostragem $C$. capitata suplantou os níveis de $A$. fraterculus diferindo das afirmações feitas por LORENZATO (1988), talvez por terem sido feitas por ele observações em outra rosácea ou cultivar, ou até mesmo utilizado outro atrativo, os quais não são mencionados em seu trabalho. Embora os números de $C$. capitata tenham sido reduzidos em relação a $A$. fraterculus é improvável a competição entre as duas espécies como foi sugerido por CHRISTENSON \& FOOTE (1960) para a Costa Rica, possivelmente pelo fato do pessegueiro não ser hospedeiro preferido pela primeira. 


\section{REFERÊNCIAS BIBLIOGRÁFICAS}

Bleicher, E.; A.L. Schroeder \& J. Bleicher. 1978. Flutuação da "moscas das frutas" (Anastrepha fratercula Wied., 1830) no município de Videira. Florianópolis, Secretaria da Agricultura e Abastecimento, 9p.

Bleicher, J.; D.N. Gassen; L.G. Ribeiro; H. TAnakA, \& A.I. Orth. 1982. Moscas-das-frutas em macieira e pessegueiro. Florianópolis, EMPASC, 28p. Bressan, S. \& M.C. TELES. 1991. Lista de hospedeiros e índices de infestação de algumas espécies do gênero Anastrepha Schiner, 1868 (Diptera, Tephritidae) na região de Ribeirão Preto - São Paulo. An. Soc. Entomol. Brasil. 20 (1): 5-15. Christenson, L.O. \& R.H. Foote. 1960. Biology of fruit flies. Ann. Rev. Entomol. (5): 171-192.

CostA, R.G. 1958. Alguns insetos e outros pequenos animais que danificam plantas cultivadas no Rio Grande do Sul. Porto Alegre, S.I.P.A., 296p.

FEHN, L.M. 1981. Coleta e reconhecimento de moscas das frutas na Região Metropolitana de Curitiba e Irati, Paraná, Brasil. An. Soc. Entomol. Brasil 10 (2): 199-208.

Gallo, D.; O. Nakano; S. Silveira Neto; R.P.L. Carvalho; G.C.D. De BatisTA; E. Berti Filho; J.R.P. Parra; R.A. ZuCChi; S.B. Alves \& J.D. VEndRAMIN. 1988. Manual de Entomologia Agrícola. São Paulo, Agronômica Ceres, 649 p.

Hickel, E.R. \& J-P. H.J. DuCRoQUET. 1993. Flutuação populacional de espécies de Anastrepha sp. (Diptera: Tephritidae) relacionadas com a fenologia de frutificação do pêssego e ameixa em Santa Catarina. An. Soc. Entomol. Brasil 22 (3): 591-593.

LorEnZATO, D. 1988. Controle integrado de moscas-das-frutas em frutíferas rosáceas. Ipagro Informa (1): 57-70.

Matioli, J.C.; M.M. Rossi \& V.H. BuENO. 1988. Suscetibilidade de cultivares de pêssego às moscas das frutas (Diptera: Tephritidae) na região de Caldas - MG. Observações preliminares. An. Soc. Entomol. Brasil. 17 (supl.): 75-85.

-1989. Atrativos para Ceratitis capitata (Wied.) em pomar de pessegueiro no município de Caldas - MG. An. Soc. Entomol. Brasil 18 (sup.): 119-129.

SALLES, L.A.B. 1991. Moscas-das-frutas Anastrepha fraterculus (Wied., 1830):

Bioecologia e controle. Pelotas, EMBRAPA/CNPFT, 16p.

. 1994. Períodos de ataque e de controle da mosca-das-frutas em pessegueiro. Horti Sul 3 (1): 47-51.

Salles, L.A.B. \& A. Kovaleski. 1990. Moscas-das-frutas em macieira e pessegueiro no Rio Grande do Sul. Horti Sul 1 (3): 5-9.

ZuCCHI, R. A.; S. Silveira Neto \& O. NAKANO.1993. Guia de Identificação de

Pragas Agrícolas. Piracicaba, FEALQ, 139p.

Recebido em 28.II.1997; aceito em 13.IV.1998. 